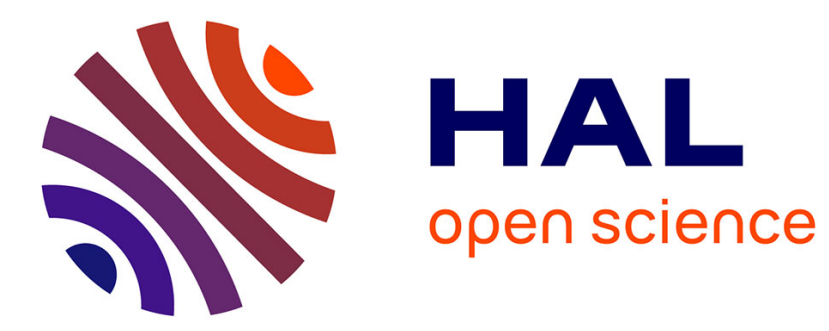

\title{
La configuration des pratiques d'information selon la logique des situations.
}

\author{
Julien Figeac
}

\section{To cite this version:}

Julien Figeac. La configuration des pratiques d'information selon la logique des situations.. Réseaux: communication, technologie, société, 2007, 25 (143), pp.17 - 44. halshs-00411579

\section{HAL Id: halshs-00411579 \\ https://shs.hal.science/halshs-00411579}

Submitted on 28 Aug 2009

HAL is a multi-disciplinary open access archive for the deposit and dissemination of scientific research documents, whether they are published or not. The documents may come from teaching and research institutions in France or abroad, or from public or private research centers.
L'archive ouverte pluridisciplinaire HAL, est destinée au dépôt et à la diffusion de documents scientifiques de niveau recherche, publiés ou non, émanant des établissements d'enseignement et de recherche français ou étrangers, des laboratoires publics ou privés. 


\title{
LA CONFIGURATION DES PRATIQUES D'INFORMATION SELON LA LOGIQUE DES SITUATIONS
}

Julien FIGEAC

Résumé :

Comment s'opère la distribution des pratiques d'information autour des usages successifs de différents supports médiatiques ? Dans cet article, nous développons l'hypothèse selon laquelle la sélection des sources d'information ne peut pas être uniquement expliquée à partir de leur légitimité culturelle et des profils sociodémographiques des individus. Nous avançons l'idée que cette sélection des supports d'information, et les usages qui en sont faits, prennent également appui sur des opportunités puisées dans les situations quotidiennes. A travers plusieurs exemples paradigmatiques, nous tentons de décrire comment ces opportunités répondent à des prises situées localement: que ce soit l'environnement ambiant, formé notamment par les médias et les TIC à disposition, ou que ce soit la possibilité d'utiliser un support en présence d'autres personnes et en conduisant d'autres activités.

\begin{abstract}
:
How are information practices distributed around successive uses of different media? In this article the author posits that the selection of information sources cannot be explained only in terms of the cultural legitimacy of that information or of individuals' sociodemographic profiles. He argues that the selection of media, and the uses to which they are put, are also based on opportunities in everyday situations. Through several paradigmatic examples, he describes how these opportunities correspond to local affordances, whether in the general environment consisting mainly of the media and ICT, or in the possibility of using a medium in the presence of other people and simultaneously doing something else.
\end{abstract}




\begin{abstract}
"Entre l'information que chacun va chercher ponctuellement, dont il maîtrise la consommation, et celle qu'il reçoit en continu, qui le submerge sans qu'il l'ait souhaité, c'est très souvent la seconde qui s'impose. Par les écrans présents un peu partout dans sa vie quotidienne, par les journaux gratuits distribués aux quatre coins de la ville, par la radio allumée en permanence. Le consommateur de médias a ainsi tendance à se nourrir d'information tout le temps, « au plus vite et au plus simple »". ${ }^{1}$
\end{abstract}

Le champ des pratiques médiatiques est profondément renouvelé par une double tendance à la multiplication et à la diversification des médias. La multiplication s'opère dans l'espace domestique par le fait qu'en parallèle des télévisions et des radios placées dans les pièces du foyer à usage collectif (les salons et les cuisines), d'autres postes sont introduits dans les pièces plus intimes comme les chambres à coucher et les salles de bain. Ces agencements des habitats contribuent à renouveler l'économie des pratiques en les désolidarisant des relations familiales ${ }^{2}$ pour permettre à chacun des membres du domicile de développer des usages davantage en phase avec ses goûts ${ }^{3}$.

La tendance à la diversification découle quant à elle du développement des TIC. La démocratisation des ordinateurs et des abonnements à l'Internet haut débit contribue, par l'étendue des activités qu'ils rendent possible, à renouveler en profondeur les habitudes médiatiques domiciliaires. Cette diversification a également un impact croissant sur les pratiques développées en situation de mobilité. A titre d'exemple, les téléphones mobiles de troisième génération $(3 \mathrm{G})$ peuvent aujourd'hui être utilisés pour regarder des chaînes télévisées ou des vidéos pendant les temps de transport.

Nous interrogeons ici une des conséquences de cette double tendance : comment les individus donnent-ils une cohérence à leurs pratiques quand elles se diversifient et se distribuent autour d'utilisations successives de différents supports médiatiques ${ }^{4}$ ? Afin d'observer ces entrelacements des pratiques autour de plusieurs médias, nous avons pris comme observatoire les informations journalistiques généralistes. Cependant, cette entrée est problématique puisque les discours journalistiques sont informés sous des formats si hétérogènes qu'il est difficile de les appréhender de manière unifiée. Par exemple, chercher à étudier la seule réception des journaux télévisés demande de mettre en place une typologie des différents " genres télévisuels d'information ${ }^{5}$ et des contextes où ils sont regardés ${ }^{6}$. Nous prenons malgré tout le parti de les réunifier sous un seul et même genre en prolongeant le sens commun qui agglomère, sous le fait de s'informer, l'ensemble des «genres » que les informations peuvent revêtir. Cette réunification va nous permettre d'investir les usages de l'ensemble des sources mobilisées par les individus pour s'informer. C'est donc en prenant cet observatoire que nous avons observé la manière dont les pratiques d'information sont entrelacées autour des usages successifs des médias de masse (la télévision, la radio et la presse écrite) et des TIC (les ordinateurs et les téléphones mobiles multimédia).

\title{
Vers une pragmatique des pratiques d'information
}

La capacité qu'ont les individus à rationaliser les entrelacements entre les supports médiatiques peut se lire au travers des stratégies de recherche d'informations, comme celles développées

\footnotetext{
${ }^{1}$ Le Monde, 23/12/04. Article synthétisant les résultats de l'étude "La mal info" (MUZET, 2006).

${ }^{2}$ MORLEY, 1986 ; PROULX, LABERGE, 1995.

${ }^{3}$ PHARABOD, 2004a, 2004b.

${ }^{4}$ PROULX, 2002.

${ }^{5}$ CHARAUDEAU, 1997a, 1997b.

${ }^{6}$ CORNER, 1991.
} 
autour de Loft Story ${ }^{7}$. Les individus passionnés par les questions d'actualité font de même. Par exemple, le lecteur de l'Equipe va faire en sorte, quand l'importance de l'événement sportif le mérite, de regarder un journal télévisé pour vivre en direct cette émotion du but que la lecture du quotidien lui a fait pressentir. Il va donc suivre l'actualité footballistique en entrelaçant la lecture du quotidien sportif avec un JT. Mais ces démarches instrumentales sont peu fréquentes. Elles sont polarisées autour de ce qui fait événement et qui, par définition, échappe au lot quotidien. Ainsi, l'intention d'utiliser un média ne répond qu'occasionnellement à de telles stratégies. Habituellement, les récepteurs butinent les gros titres afin d'actualiser leurs connaissances globales de l'actualité ${ }^{8}$.

Il apparaît ainsi que la posture habituelle des consommateurs d'information est orientée vers un horizon d'attente relativement flou. Cet horizon peut se lire dans le propos de cette femme qui regarde chaque soir, avec son mari, le 19/20 sur France 3 "parce qu'on voit un peu ce qui se passe dans la région ». De même, ce jeune homme célibataire résume bien son rapport aux informations:

E. F. : "Parfois M6 parce que c'est des concentrés d'informations. J'aime pas trop quand ça dure une demi-heure, trois quarts d'heure avec les commentaires de l'un, de l'autre, on brasse beaucoup... Je suis pas porté sur l'information à outrance, l'important, c'est de savoir ce qui s'est passé. Je trouve que Canal+ présente bien l'info comme ça, ça part pas dans des interprétations, les commentaires de l'un et ça évite d'être... pas manipulé mais trop influencé."

Ce qui compte pour lui, c'est d'avoir un accès rapide à des informations factuelles qui synthétisent les principaux faits du jour. L'essentiel étant de se tenir informé sur «ce qui s'est passé ». Si cet horizon d'attente relativement vague est premier, il peut être couplé à des visées plus précises. C'est ainsi qu'un homme d'une cinquantaine d'années achète tous les jours $L e$ Monde car son activité d'agent immobilier l'incite à veiller sur l'évolution des cotations boursières. Mais au-delà de cette motivation spécifique, il maintient avant tout cette habitude pour rester informé sur l'actualité générale. C'est dans le même esprit qu'il va prolonger sa lecture matinale par la visite du site de TF1. A travers cet usage, il cherche à coller aux derniers soubresauts de la bourse. Mais là encore, cette connexion prend tout sens au travers des rapides coups d'œil qu'il va jeter aux news de dernière minute :

P. W. : "Moi, j'aime bien me mettre sur le site TF1. J'ai les informations à la minute, des flashs qui passent. Si je veux un petit peu un article de fond, je me connecte dessus, ils développent un peu l'article. Je regarde la bourse, hop, ça va tout de suite. Et puis, je regarde la page sport. Donc, ben je sais que je peux le regarder tout le temps."

La relation aux questions d'actualité s'inscrit donc constamment sous cette tension dialectique entre les visées stratégiques, de la recherche de compléments d'information, et un horizon d'attente indéfini sur ce qui fait événement à l'instant $T$. Nous défendrons l'hypothèse que l'ouverture sur cet horizon d'attente est première mais, au gré des circonstances, selon la pertinence des événements, les pratiques peuvent être polarisées vers la recherche d'informations précises.

Le recours à des entretiens nous a permis de mieux cerner cette posture des récepteurs en leur demandant de décrire leur rapport aux informations journalistiques sous toutes leurs déclinaisons possibles. Plusieurs gros consommateurs d'information expliquent par exemple que chaque jour ils se préparent en écoutant la radio, ils lisent ensuite un quotidien dans les transports, puis consultent des news sur Internet et regardent un journal télévisé au moment du dîner. Il apparaît alors que les seules prises qui leur permettent de classifier la diversité de leurs pratiques sont les situations quotidiennes où ils entrent en relation avec les discours journalistiques. La faible continuité entre leurs usages successifs des différentes sources cède alors le pas à des motivations

\footnotetext{
${ }^{7}$ BEAUDOUIN, BEAUVISAGE, CARDON, VELKOVSKA, 2003.

${ }^{8}$ MUZET, 2006.
} 
situées localement, comme celles consistant à passer le temps ou à créer un bruit de fond pour rendre la situation plus conviviale.

Nous tenterons de démontrer que c'est parce que les individus sont en situation de pouvoir, voir de devoir, passer le temps qu'ils s'ouvrent régulièrement à l'horizon relativement flou des questions d'actualité. En effet, l'activation des pratiques d'information est souvent enchâssée dans les situations vécues : prendre le métro, être devant son poste de travail, dîner en famille, etc. Ainsi, l'intention d'utiliser un support d'information ne répond pas tant à des stratégies qu'à la configuration des situations qui rend propice son utilisation. Nous défendons donc l'hypothèse que la propension à activer les pratiques d'information est à appréhender de manière endogène entre l'horizon intentionnel des individus et la configuration des situations quotidiennes.

David Morley a posé les bases d'une telle approche en s'intéressant tout d'abord au «contexte d'écoute » de la télévision dans la cellule familiale ${ }^{9}$, puis en abordant l'inscription des supports d'information et de communication dans les foyers ${ }^{10}$. S'il s'est intéressé à la dimension des routines quotidiennes qui cadrent les « contextes d'écoute », il a avant tout fondé ses analyses sur les interactions développées par les membres de la famille autour du petit écran. Si ces deux dimensions restent incontournables pour développer une approche ethnographique sur les contextes de réception des JT, elles s'appliquent plus difficilement aux usages des autres supports d'information. En effet, dans la plupart des cas, quelqu'un qui s'informe le fait en silence quand il est seul. Si l'on croise les diverses confrontations aux discours journalistiques, il apparaît qu'elles se déroulent souvent au sein d'interactions d' " inattention civile ${ }^{11} »$ non fondées sur des activités communicationnelles. Dès lors, même si il est pertinent de chercher à reconstituer les modes de réception et l'ancrage contextuel des usages autour des échanges verbaux entre les co-récepteurs ${ }^{12}$, il n'est pas possible d'adopter ici cette entrée.

Pour aborder ces usages solitaires et silencieux, il est intéressant de s'appuyer sur les théories de l'action située. Elles permettent tout d'abord d'assimiler les plans d'action, telles que les stratégies de recherche d'information, à de simples ressources orientant les usages ${ }^{13}$. Elles donnent également la possibilité de référer l'activation des usages et leur déroulement aux multiples prises locales ${ }^{14}$, et notamment aux agencements spatiaux ${ }^{15}$, sur lesquels les individus prennent appui. Dans cette optique, nous allons proposer une lecture pragmatique de l'activité de réception des informations en montrant comment les différentes phases qui la composent, que ce soit l'intention d'utiliser un support, le choix de ce support et les usages qui en sont faits, sont codéterminées par l'individu et les prises des situations quotidiennes. Ces prises sont formées par l'environnement ambiant (le domicile, le lieu de travail, les stations de métro...) et par les médias ou les TIC à disposition, mais aussi par les activités réalisées en parallèle des usages (préparer le dîner,...) et par les individus en présence (conjoint, collègue,...). Nous tenterons de montrer comment, au cours des différentes phases de l'activité de réception, ces prises sont saisies comme pertinentes pour en orienter et en structurer la conduite.

\section{Eléments de méthodologie}

Dans cette perspective, nous avons réalisé une étude auprès de 42 individus qui ont été recrutés en raison de la régularité et de la diversité de leurs usages des supports d'information. Comme notre objectif était de sonder des pratiques émergentes, nous avons choisi de constituer un échantillon spécifique, composé pour l'essentiel d'utilisateurs "avancés" : ils font partie des foyers multiéquipés en TIC et utilisent leur téléphone mobile pour consulter des contenus multimédia. Dès

\footnotetext{
${ }^{9}$ MORLEY, 1986.

${ }^{10}$ MORLEY, SILVERSTONE, 1990.

${ }^{11}$ LICOPPE, INADA, 2005.

${ }^{12}$ RELIEU, OLSZEWSKA, 2004.

${ }^{13}$ SUCHMAN, 1987.

${ }^{14}$ QUÉRÉ, 1999.

${ }^{15}$ CONEIN, JACOPIN, 1994 ; KIRSH, 1995.
} 
lors, les individus interrogés sont majoritairement des technophiles, c'est à dire des jeunes hommes, diplômés du supérieur, qui exercent une activité professionnelle dans une grande agglomération ${ }^{16}$.

Leurs pratiques d'information ont été appréhendées à partir de questionnaires, d'entretiens et des observations ethnographiques qui ont parfois été couplées à des enregistrements vidéo. Au cours des entretiens, nous leur avons demandé de décrire leurs usages des différents supports d'information. Pour faciliter leur effort de verbalisation, et pour que leur exposé soit le plus complet possible, il s'est avéré pertinent de réaliser ces entretiens in situ face à chaque support. Nous les avons ainsi amenés à décrire sur quel(s) élément(s) des situations quotidiennes ils s'appuient pour déployer leurs usages. Les observations ethnographiques, telles que nous les avons pratiquées ${ }^{17}$, ont donc consisté à faire le tour des domiciles en demandant aux enquêtés : Pourquoi ont-ils situés chaque support d'information à cet endroit? A quel moment l'utilisent-ils ? En présence de qui se trouvent-ils lors de ces utilisations ? Font-ils une autre activité en parallèle de ces usages?

Si les données qui vont être présentées ont avant tout été recueillies à l'aide des entretiens, il s'est avéré nécessaire de recourir à des questionnaires pour récolter des informations de cadrage difficiles à obtenir à l'oral. Sans avoir été formulés pour donner lieu à un traitement statistique, ces questionnaires ont pour seule ambition de permettre de sonder avec plus de facilité les usages de l'ensemble des médias (quotidiens lus, émissions de radio écoutées, journaux télévisés regardés...) tout en répertoriant les fréquences et les lieux. Grâce à eux, nous avons pu croiser la fréquence selon laquelle les informations journalistiques sont consultées en comparaison aux autres contenus. Par exemple, cette mise à plat des pratiques permet de repérer dans quelle mesure la consultation des grilles de résultats sportifs, des bulletins météo, des programmes TV, des infos trafic ou des horoscopes peut être plus courante que la consultation des informations les plus légitimes.

\section{COMBINER LES PRATIQUES D'INFORMATION AVEC LA LOGIQUE DES SITUATIONS}

Dès lors que l'on prête attention aux situations, il devient possible de lier l'audience massive des journaux télévisés et radiodiffusés à la possibilité d'articuler leur écoute à un grand éventail de tâches quotidiennes, comme à une grande variété d'interactions. De même, en ce qui concerne la presse écrite, la substitution des gratuits aux achats de quotidiens payants peut ne plus être réduite au seul critère de gratuité. Elle peut être renvoyée à ces prises situées localement que forment les distributeurs de journaux gratuits au carrefour des situations de mobilité. Quant aux usages des sites de presse, il devient possible de les situer au carrefour des multiples activités pouvant être réalisées à partir des ordinateurs connectés à l'Internet haut débit. Enfin, nous nous intéresserons aux usages émergents des services multimédia consultables depuis les téléphones mobiles de troisième génération. Il s'agira de montrer comment le recours à ces services est associé à la vaste gamme des pratiques d'information dans la mesure où les téléphones mobiles, en supports médiatiques qui tiennent dans les poches, forment de nouvelles prises pour meubler certaines situations par la consultation des news.

\section{Les audiences massives de la télévision et de la radio : entre le registre de la multi-activité et les types d'interactions}

Les journaux télévisés constituent une véritable institution. La plupart des individus interrogés les regardent tous les jours ou presque. Nous avons cherché à comprendre leur succès à travers la perspective générale de cette étude qui vise à caractériser ce qui différencie les JT des autres

\footnotetext{
${ }^{16}$ Dans le cadre de cette étude, 28 des personnes interrogées vivent dans la région parisienne et 14 résident à Toulouse ou dans sa périphérie.

${ }^{17}$ PHARABOD, 2004a.
} 
sources d'information. Si les JT restent au cœur des pratiques, n'est-ce pas en raison de leur grande capacité à se marier à la vie des foyers ? Et le succès des informations radiodiffusées, comme celui des JT, ne découlerait-il pas de la possibilité d'articuler leur réception avec de nombreuses formes d'interactions ainsi qu'avec la réalisation de nombreuses activités?

\section{Les usages de la radio : entre la configuration des situations et le registre de la multi-activité}

Pourquoi utiliser la radio pour s'informer ? Nous répondrons à cette question en montrant que la radio reste ancrée dans les habitudes médiatiques dans la mesure où elle est "le" média de la multi-activité : elle s'écoute en voiture, pendant les préparatifs du matin ou, d'une manière ou d'une autre, pendant que l'on fait autre chose.

Parmi les individus interrogés, ceux qui écoutent le plus la radio sont ceux qui utilisent leur voiture pour se rendre sur leur lieu de travail. Le fait de devoir prendre régulièrement le volant les conduit à développer les goûts radiophoniques les plus prononcés et les plus éclectiques. Cette situation de conduite joue un rôle actif dans la configuration de leurs pratiques radiophoniques dans la mesure où le poste de radio forme une opportunité de divertissement. Plus un individu passe de temps dans sa voiture, plus le caractère contraignant, voire rébarbatif, de la situation va l'inciter à l'utiliser. C'est ainsi qu' au fil de sa conduite, le passionné de musique pourra éprouver l'envie de changer de registre en écoutant les nouvelles du jour. Par exemple, ce jeune homme privilégie l'écoute des stations musicales mais il apprécie que la programmation soit entrecoupée de bulletins d'information :

M. T. : "Ça permet quand même de se tenir au courant de l'actualité et puis ben d'avoir un peu des avis différents, d'avoir des auditeurs, enfin d'écouter les auditeurs qui peuvent donner leur avis sur les évènements, d'avoir le nombre de voitures brûlées pendant la nuit... Être au courant de l'actualité en fait, en quelques minutes, avant d'arriver au boulot sans avoir forcément besoin de s'acheter les journaux, quoique maintenant on les achète plus parce qu'il y a des journaux gratuits."

Ainsi, ce sont ceux qui s'engagent régulièrement dans des situations propices à l'écoute de ce média qui diversifient le plus leurs usages et finissent par le mobiliser comme une source d'information à part entière.

$\mathrm{Au}$ domicile, la compagnie des animateurs peut également être très appréciée, notamment pendant la réalisation des activités fastidieuses telles que les tâches ménagères ou les démarches administratives. Leurs discours peuvent alors être écoutés d'une seule oreille pendant que l'attention est polarisée sur le déroulement de l'activité principale. La réception de la radio repose souvent sur une attention de second degré qui entretient une relation, plus ou moins étroite suivant les circonstances, avec le premier degré d'attention. L'essentiel dans l'écoute de la radio n'est donc pas nécessairement l'écoute elle-même. Elle permet avant tout de créer un bruit de fond auquel on va accorder des degrés d'attention variables. Cette modalité de réception, oscillant entre des degrés d'écoute différents, caractérise un mode de divertissement qui a pour finalité de rendre la réalisation de l'activité principale moins fastidieuse ${ }^{18}$.

Ce type d'engagement dans la réception fait que la radio est peu propice à la recherche d'informations précises. Elle s'écoute selon une logique de flux ${ }^{19}$, par intermittence, tout en étant souvent subordonnée aux tâches quotidiennes. Ainsi, sur le même registre que les postes de radio équipant les voitures, la réalisation de certaines activités, telles que les tâches ménagères, constituent des opportunités d'usages que vont saisir les auditeurs. C'est à partir de la fréquence des opportunités saisies que va se réaliser la dynamique des goûts radiophoniques : plus la fréquence sera régulière, plus la radio constituera une source d'information à part entière.

\footnotetext{
${ }^{18}$ CERTEAU, 1980.

${ }^{19}$ SEMPRINI, 1995.
} 


\section{Les modes de réception des journaux télévisés : entre les types d'interactions et les tâches quotidiennes}

Au cours de cette partie, sur l'inscription des journaux télévisés dans les pratiques d'information, nous n'aborderons pas de front le décodage des JT. Nous ferons glisser le regard du côté de leur « contexte d'écoute ${ }^{20} »$. David Morley a largement balisé cette perspective en montrant comment le «contexte d'écoute » naturel de la télévision, la sphère domestique, influe sur la sélection des programmes et sur leur réception. Pour lui, cette influence se joue tout d'abord par le biais des activités communicationnelles que les membres du foyer développent autour du petit écran. Elle se joue également au niveau des routines domestiques qui accompagnent l'activité de réception. Nous souhaitons ici prolonger ces deux versants en nous focalisant sur les «contextes d'écoute » des journaux télévisés à travers la perspective générale de cette étude qui tend à caractériser comment, en fonction de la configuration des situations, un individu va décider de mobiliser un support d'information plutôt qu'un autre. Ainsi, nous allons tenter d'évaluer pourquoi, alors que de multiples supports sont disponibles dans la sphère domestique, les individus interrogés vont privilégier l'écoute des journaux télévisés.

Taylor et Harper $^{21}$ ont montré que le degré d'engagement dans l'écoute de la télévision varie suivant les rythmes de la vie du foyer. En ce qui concerne l'heure à laquelle les JT sont regardés, elle concorde avec une phase de la vie de famille riche en activités. Leur écoute est souvent doublement secondaire. Elle passe après les interactions entre les membres du foyer et les discussions qu'ils peuvent entretenir. Et elle est subordonnée aux repas et à leurs préparatifs. De ce fait, la réception des JT est entièrement en prise avec l'ensemble des interactions et des activités se déroulant dans ce temps particulier de la vie familiale. Par rapport aux autres supports d'information disponibles dans l'espace domestique, leur particularité tient à la possibilité d'articuler facilement leur écoute avec ces deux dimensions. Pour caractériser cette tendance, nous allons montrer comment les situations de réception des JT fluctuent selon la situation matrimoniale.

Ceux qui vivent maritalement vont développer des «arts de faire ${ }^{22}$ consistant à conjuguer l'écoute des JT avec les interactions de la vie de couple (ou de la vie de famille). Comme il est quasiment impossible d'observer ces ajustements in situ, nous avons pu prendre conscience de ces modes de réception à l'aide des entretiens effectués auprès des jeunes couples :

J.M.F. : "(...) disons qu'on mange, on mange devant le JT, mais on le laisse en fond histoire de, bon c'est... histoire d'avoir un petit fond sonore. Quand on mange, quand on est là, on n'est pas non plus scotché devant la télé. (...). Si on parle et qu'il y a quelque chose, un sujet intéressant, bon, ben, on va s'arrêter de parler, on va regarder ce qui nous intéresse."

Les jeunes couples verbalisent facilement cette dimension car, pour eux, l'écoute de l'autre est revendiquée comme centrale. Ils disent avoir réfléchi à la place qu'occupe la télévision dans leur vie et ont fait le choix de lui faire jouer un rôle secondaire pour qu'elle ne vienne pas se substituer à leurs discussions. Mais ce n'est pas parce qu'ils privilégient leurs échanges qu'ils vont s'interdire de regarder un JT puisque, en le réduisant à un bruit de fond, ils cherchent à rendre l'ambiance plus agréable. Ils réduisent alors les discours du présentateur à un rôle de fond sonore qui viendra combler les phases de silence ou qui fournira des prises pour relancer les discussions.

Ils développent ainsi des formes d'écoute consistant à accorder une oreille aux proches et l'autre au présentateur du JT. Leur attention va être distribuée en fonction de l'importance du sujet traité. S'il le trouve peu important, le récepteur va accorder la majeure partie de son attention à ses proches. Mais, lorsque le présentateur va évoquer un sujet intéressant, il va détourner son écoute des échanges familiaux pour réinvestir le discours du journaliste. Si cette écoute distribuée peut susciter des tensions, dans la mesure où celui qui avait la parole peut considérer que le

\footnotetext{
${ }^{20}$ MORLEY, 1986.

${ }^{21}$ TAYLOR, HARPER, 2003.

22 DE CERTEAU, 1980.
} 
téléspectateur expert qui partage sa vie ne l'écoute que d'une oreille, il se forme en général un équilibre autour du partage de cette expertise. Il y a équilibre car l'ensemble des individus qui discutent entre eux accordent tous une partie de leur attention au JT.

Ces modes de réception, développés par les jeunes couples, ne sont pas transposables dans toutes leurs singularités aux autres classes d'âge :

LVH : "Je suis pas comme disons... des gens de 40, 50 ans disons, qui ont toujours été habitués à regarder le journal télé... à huit heures, c'était allumé le journal télé."

On peut quand même observer que, même chez les couples qui ont des habitudes médiatiques davantage stabilisées, la manière dont l'écoute des JT va s'articuler avec les dîners et leurs préparatifs n'est pas homogène : elle va osciller entre des degrés d'attention différents. Ces degrés fluctuent entre un engagement fort ${ }^{23}$, quand les téléspectateurs ne font rien d'autre que regarder le programme, et des niveaux où l'activité de réception est à la fois subordonnée aux interactions avec les individus en présence et aux tâches effectuées autour du petit écran.

Comme les individus vivant en couple, les célibataires se servent souvent des JT pour créer une présence. Mais, la solitude de leurs usages a de lourdes répercussions sur leur choix de programmes ainsi que la manière de les regarder. Une fois devant leur écran, leur engagement dans l'activité de réception est beaucoup plus intense. Dans la mesure où ils peuvent facilement passer d'un programme à l'autre, sans que cela dérange un quelconque co-récepteur, ils utilisent fréquemment leur télécommande pour naviguer entre les différentes éditions. Cette activité de "zapping" reste indissociable de l'activité réflexive qu'ils portent sur les JT dans la mesure où, c'est en comparant la manière dont les différentes éditions vont traiter les sujets, qu'ils vont se forger leur propre opinion.

A travers la relation que les célibataires et les personnes vivant en couple entretiennent avec les JT, nous prolongeons l'idée défendue par Morley selon laquelle leur réception est toujours à référer aux interactions familiales - ou à leur absence - ainsi qu'aux activités développées autour du petit écran. Elle invite à aborder le choix des programmes télévisés comme une délibération faite en appui avec la configuration des situations d'usage. Le format des JT a la particularité de coïncider parfaitement avec l'effervescence des activités liées aux repas. C'est pourquoi les autres supports d'information domiciliaires, dont les usages peuvent être plus isolants et moins propices à la multiactivité, ne sauraient concurrencer les journaux télévisés durant cette phase de la vie domestique.

\section{L'engouement pour la presse gratuite : entre l'argument de la gratuité et l'opportunité en situation de mobilité}

Comme le montre le schéma ci-dessous, les individus interrogés dans le cadre de cette étude s'informent régulièrement à travers la presse écrite. Cependant, s'ils reconnaissent la grande légitimité des quotidiens "payants", ils n'en font pas une source d'information privilégiée dans la mesure où ils lisent quasi-exclusivement la presse urbaine gratuite.

\footnotetext{
${ }^{23}$ Il est difficile de mesurer le degré d'attention qu'un téléspectateur accorde à un programme. LULL (1990) déduit l'intensité avec laquelle un téléspectateur s'investit dans la réception d'un programme du plaisir qu'il en retire.
} 


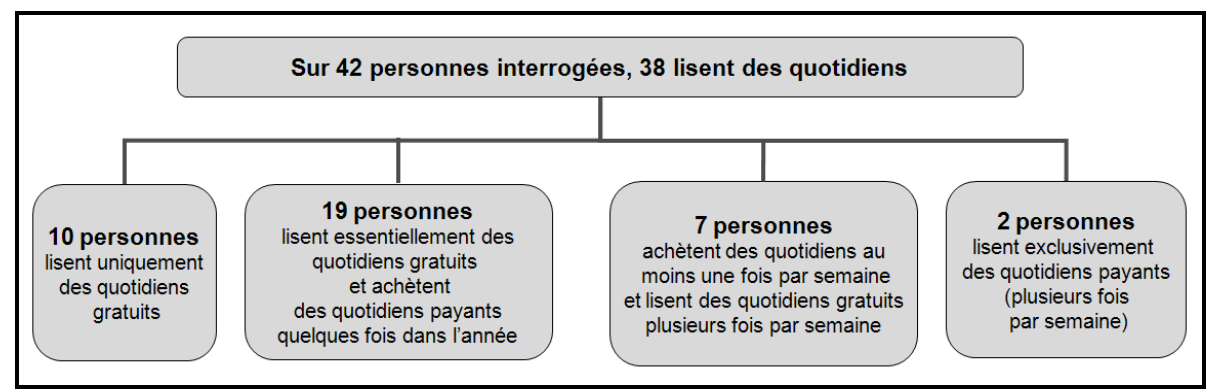

Répartition des pratiques observées autour de la presse "payante" et gratuite

Le principal argument évoqué, pour justifier ce désaveu, est d'ordre économique. On l'observe plus particulièrement chez les jeunes, étudiants ou actifs, qui expliquent que leur situation financière ne leur permet pas d'en acheter chaque jour un exemplaire :

"Moi, j'achète pas les journaux en général le matin parce que c'est un vrai budget. Donc, si je prends des journaux, c'est des journaux gratuits."

Pour lire leurs éditions de référence tout en faisant l'économie du détour par les kiosques, certains disent exploiter les multiples opportunités qui se présentent à eux. Par exemple, les cadres interrogés consultent régulièrement les quotidiens qui sont mis à leur disposition sur leur lieu de travail. Certains employés profitent quant à eux des détours par la machine à café pour essayer de capter l'édition d'un collègue. Et on trouve enfin les amateurs de sports qui préfèrent faire un détour par le café du coin, plutôt que par un kiosque, pour consulter les grilles sportives.

Il n'est pas possible de réduire ces usages opportunistes à des stratégies, orientées vers la seule économie des achats de quotidiens, car ces individus se contentent de profiter des occasions de lecture qui se présentent à eux. Cette tendance montre que ceux qui lisent les quotidiens mis à disposition par leur entreprise, leurs collègues ou les cafés, ne les ont pas nécessairement sélectionnés en fonction de leur coloration politique, la qualité de leurs analyses, etc. Dans ces cas là, l'opportunité de lecture prend le pas sur la dynamique des goûts personnels. Et, c'est en fonction de ces multiples opportunités, puisées également dans les salles d'attentes ou les zones d'embarcation des aéroports, que la stabilité des goûts est renouvelée.

Si cette tendance peut paraitre anecdotique, elle prend une toute autre ampleur quand on la croise avec la relation aux quotidiens gratuits. Si la plupart des individus interrogés lisent des gratuits tous les jours ou presque, ils ne font pas le calcul de contourner les achats de quotidiens puisque, de toute façon, ils n'avaient pas nécessairement pris l'habitude de les acheter. Il n'est pas possible de réduire le succès des gratuits au seul argument économique. Il repose aussi sur le fait qu'ils viennent vers les lecteurs en étant distribués, par des humains et des non-humains, dans des points de passage très fréquentés comme l'entrée des bouches de métro. Le fait que ces distributeurs se trouvent aux carrefours des trajectoires urbaines permet à ceux qui ne sont pas prêts à payer le "prix" de la lecture des "payants", de lire malgré tout la presse. L'économie qu'ils réalisent n'est pas uniquement pécuniaire. Ils font aussi celle du coût temporel nécessaire au détour par un kiosque, du coût physique nécessaire pour l'atteindre, du coût cognitif nécessaire pour ne pas oublier de faire ce détour et, le cas échéant, du coût psychologique découlant de la frustration d'avoir oublié de faire ce détour.

Il apparaît donc que le détour par les situations quotidiennes permet d'expliquer pourquoi cette presse, peu valorisée socialement, rencontre un tel engouement. La valeur de ces quotidiens n'est pas à chercher dans leur format, ni dans la qualité des informations qu'ils diffusent. Elle émane de la logique des situations qui fait, qu'à l'endroit précis et au moment précis où un passant va tomber sur ce quotidien, il va lui attribuer une grande valeur, une valeur circonstancielle, celle de l'occuper l'espace de cinq minutes. De plus, la valeur de ces quotidiens ne peut pas être évaluée sans être réinscrite dans la globalité des pratiques d'informations. Les utilisateurs "avancés" 
étudiés ici se tournent vers eux sans complexe au regard de leurs multiples confrontations aux discours journalistiques.

Les usages de la presse en ligne : entre le design des interfaces et la séquentialisation des activités

Même si dans notre échantillon les jeunes technophiles sont surreprésentés, les individus interrogés mobilisent rarement Internet pour rechercher des informations journalistiques. Dans la mesure où ils peuvent les consulter à partir des autres médias, ils vont profiter d'Internet pour développer d'autres activités. Ils vont s'en servir pour communiquer par le biais des messageries synchrones ou asynchrones. Et, s'ils doivent l'utiliser pour rechercher des informations, ils s'orienteront plutôt vers des informations pratiques (sur des biens de consommations, sur les horaires de transport, etc.) et des informations spécialisées (en informatique, en cuisine, etc.).

Si l'on se situe du côté des usages, il est difficile d'attester l'idée selon laquelle Internet absorberait les autres médias. En effet, les individus les plus férus d'un titre de quotidien, d'une émission de télévision ou de radio, utilisent peu Internet pour aller visiter les portails qui leurs sont dédiés. Dans la mesure où la diversité des contenus accessibles depuis un ordinateur va bien au-delà de ceux proposés par les autres médias, ils s'en servent pour explorer de nouveaux horizons, comme les sites qui proposent des vidéos en streaming (YouTube, Dailymotion,...), plutôt que pour approfondir ceux qui sont banalisés dans leurs habitudes médiatiques.

Le cas des informations journalistiques est particulièrement révélateur de cette tendance puisque les ressources, et les contraintes propres aux modalités de navigation sur Internet, vont inciter les utilisateurs à s'informer à partir de nouvelles sources. Les rares fois où les individus interrogés s'informent à partir d'Internet, ils se tournent quasi-exclusivement vers les "News" diffusées sur la page d'accueil de leur F.A.I. (Fournisseur d'Accès Internet), celle de l'hébergeur de leur compte de messagerie électronique (Yahoo, Voila, etc.) ou celle de leur moteur de recherche (Google News). Nous savons que "l'étude des parcours (sur Internet) est donc indissociable de l'étude des productions de la toile, à la fois pour donner sens aux parcours et pour montrer comment la structure et les contenus des sites agissent sur les visites » ${ }^{24}$. Dès lors, pour expliquer l'audience de ces pages, il convient de comprendre comment les usagers y accèdent.

\section{Le rôle du design des interfaces sur la consultation des "News"}

Les individus interrogés ont tendance à garder en page d'accueil de leur navigateur Internet celle de leur F.A.I. ${ }^{25}$. Par exemple, les abonnés à Orange conservent souvent le portail d'Orange en page d'accueil de leur navigateur. Maintenant que l'on sait que ces pages s'invitent au regard de ces internautes, il convient de s'intéresser à leur mise en forme afin d'observer la place accordée à la rubrique "News" (Cf. ci-dessous la Page d'accueil du site du groupe Orange).

\footnotetext{
${ }^{24}$ BEAUDOUIN, FLEURY, PASQUIER, HABERT, LICOPPE, 2002.

${ }^{25}$ Cette configuration se fait souvent par défaut lors de l'installation du modem.
} 


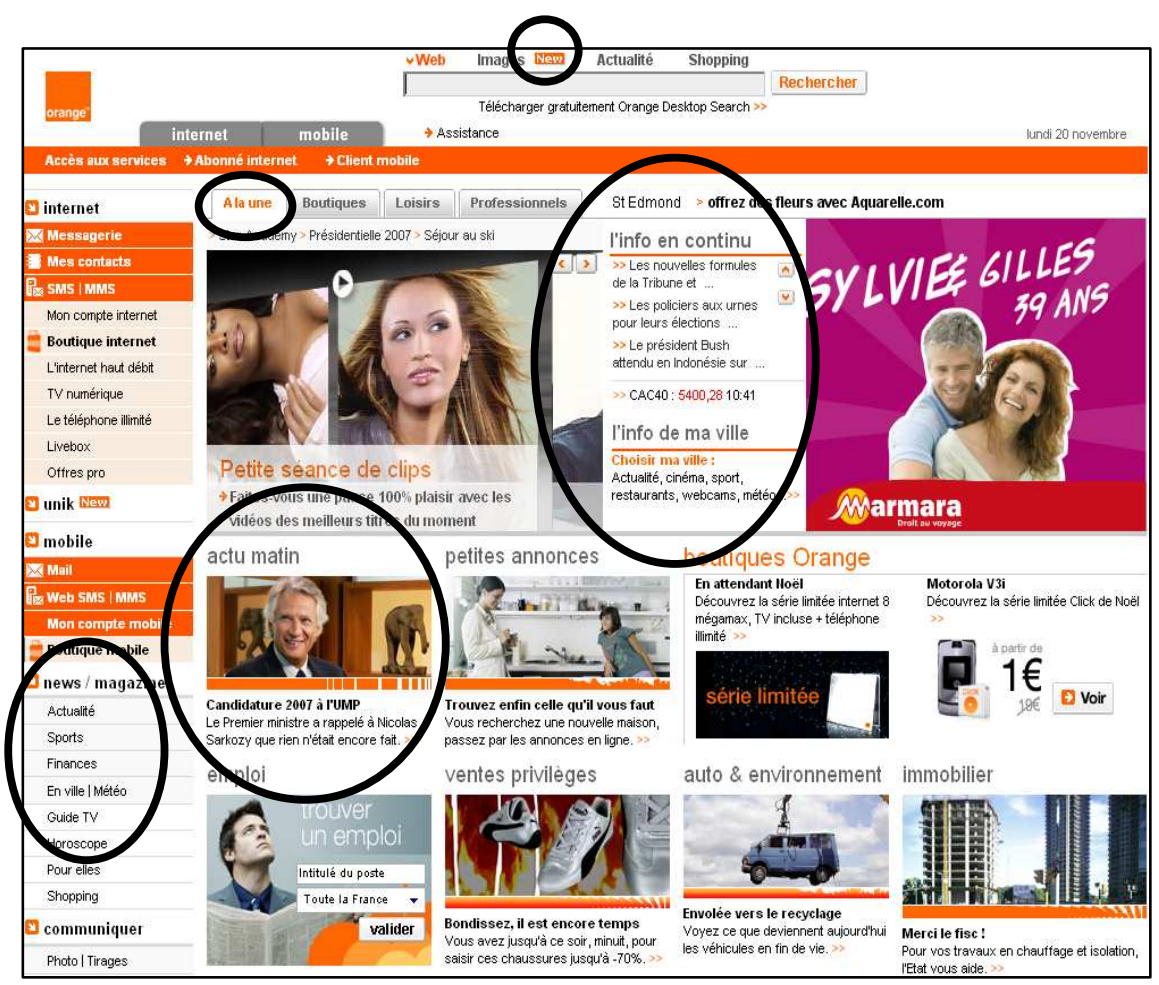

Page d'accueil du site du groupe Orange

Cette capture d'écran montre comment, sur la page d'accueil du site d'Orange, ont été intégrés différents accès aux "News" autour des liens dédiés à l'activité commerciale de l'opérateur (ceux en haut à gauche de l'écran). La configuration de la page d'accueil de ce F.A.I. ne lui est pas spécifique, elle a une structure proche de celles de ses concurrents ${ }^{26}$. Dès lors, dans la mesure où les navigateurs Internet s'ouvrent fréquemment sur ces pages, il convient de référer la place qu'occupent les news dans les pratiques observées, à leur inscription dans les modalités de navigation. Nous retrouvons ici un type d'accès à l'information qui nous semble paradigmatique. Il renforce l'idée selon laquelle ces usages ne peuvent pas être interprétés sous les seuls aspects des profils des individus et de la légitimité des sources, tant ces prises locales influent sur la sélection des sources.

\section{Resituer la consultation des "News" dans la séquentialisation des activités}

L'autre dimension qui permet de comprendre l'audience de ces rubriques est l'enchaînement des activités réalisées à partir d'un ordinateur et des connexions Internet. Si, comme nous l'avons dit, les individus interrogés se servent davantage d'Internet pour développer de nouvelles formes de communication, plutôt que pour rechercher des informations journalistiques, il faut considérer que ces activités ne sont pas toujours indépendantes. Elles forment un même territoire de navigations : le territoire $«$ familier ${ }^{27}$.

Les individus qui souhaitent consulter leur boîte aux lettres électroniques personnelle, notamment quand ils sont au travail et qu'ils n'ont pas de logiciel de messagerie, doivent s'identifier sur l'interface Webmail de leur hébergeur. Or, ces hébergeurs, qu'ils soient des F.A.I. ou non (Yahoo, Voila, etc.), proposent des rubriques "News". Dès lors, dans la mesure où pour consulter leurs

\footnotetext{
${ }^{26} \mathrm{Si}$ une personne qui n'est pas abonnée à un F.A.I. se rend sur sa page d'accueil, elle tombera souvent sur des liens promotionnels. Ce n'est qu'une fois qu'elle sera abonnée que la page d'accueil du F.A.I. proposera des contenus, dont des «News».

${ }^{27}$ BEAUVISAGE, 2004.
} 
courriers électroniques, les internautes doivent passer par la page d'accueil de leur hébergeur, ils sont d'autant plus incités à faire un détour par ces rubriques.

Sur le même registre, d'autres individus disent s'informer régulièrement à partir de la rubrique "Actualités» du moteur de recherche Google. Là encore, la consultation de cette rubrique bénéficie, en tant qu'activité secondaire, de l'aura de l'activité principale qu'est la recherche d'informations au sens large.

Si les consultations Internet des informations journalistiques répondent à deux types de prises, celle du design de l'interface et celle de la séquentialisation des activités, il ne faut pas pour autant minimiser la dimension planifiée et stratégique de certains usages. Il arrive que les individus mobilisent Internet pour rechercher des compléments d'informations. Dans ces cas là, ils vont planifier leur navigation pour atteindre leur but. Mais l'erreur serait de croire que la valeur de l'information recherchée tient nécessairement à la légitimité des analyses développées par les journalistes. Elle tient davantage à leur caractère synthétique et factuel qui va en optimiser la recherche. Là encore, les rubriques "News" sont privilégiées dans la mesure où elles listent de manière claire et hiérarchisée des dépêches gratuites, factuelles et renouvelées en continu. En ce sens, la mobilisation d'Internet prolonge la méfiance envers les discours de presse qui, par la longueur et la complexité de leurs analyses, éveillent un sentiment de suspicion. En lecteurs éclairés, qui savent peser le pour et le contre, ils disent privilégier les informations factuelles qui se contentent de rapporter les événements sans chercher à les mettre en perspective.

Cependant, il ne faut pas généraliser cette tendance. Elle est bien spécifique aux utilisateurs "avancés" qui déclinent leurs pratiques d'information sur plusieurs supports. Dans la mesure où ils entretiennent une relation étroite avec les questions d'actualité, ils orientent leurs consultations Internet vers d'autres types de contenus comme les informations pratiques et spécialisées. Autrement dit, ils profitent de la solitude de leurs usages d'Internet pour s'ouvrir à de nouvelles activités et à de nouvelles informations, là où les usages collectifs des autres médias constituent des occasions plus propices à l'écoute des informations journalistiques.

\section{Les services 3G : entre la disponibilité des téléphones mobiles et l'accessibilité des news}

Nous avons également cherché à comprendre comment les services multimédias, consultables depuis les téléphones mobiles de troisième génération $(3 \mathrm{G})$, peuvent être associés aux pratiques d'information. Dès lors, parmi la diversité des contenus proposés par ces services, un focus a été opéré sur ceux. 


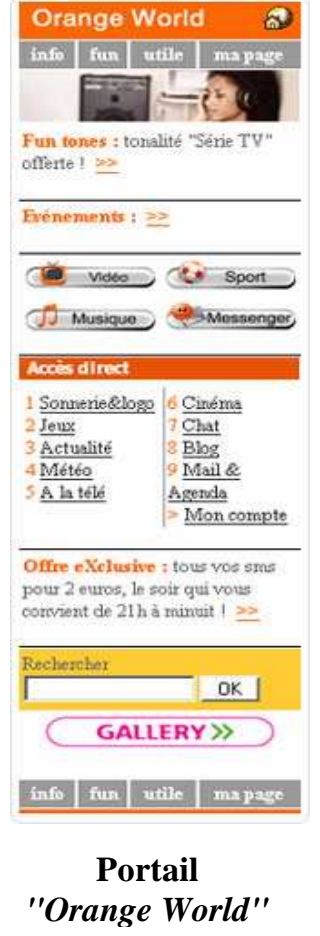

Les portails "Orange World" et "Vodafone live!" (SFR) proposent trois types de contenus :

- Le WAP : Le Wireless Application Protocol est un protocole de communication qui permet d'accéder à Internet à l'aide d'un téléphone portable. Cet accès à Internet déborde difficilement des pages de contenus proposées sur les portails des opérateurs (cf. ci-contre la capture d'écran du portail "'Orange World"). Nous avons observé les usages du WAP orientés vers la consultation des informations généralistes, c'est-à-dire les rubriques "News", "Sports" et "Météo". Chacune de ces rubriques diffusent des dépêches d'agences, des images de l'actualité et des vidéos.

- Les vidéos en streaming: l'opérateur Orange donne accès, sur son service "Orange World", à des flashs infos de deux minutes condensant les journaux télévisés de iTélé ainsi qu'un bulletin météo, des reportages, le journal du foot et le journal des sports (également produits par les journalistes de iTélé). Ces vidéos, comme les chaînes TV, sont consultables en streaming, c'est-à-dire en temps réel sans nécessiter de téléchargement.

- Les chaînes télévisées : les abonnés Orange ayant souscrit à l' « Option TV » (6€ / mois) bénéficie d'un accès illimité à 20 chaînes télévisées. Nous nous sommes avant tout intéressés à la consultation des JT, des chaînes d'information en continu (LCI, BFM TV, ...) et des programmes entrecoupés par des bulletins d'information (Télématin,...)

Si les dépêches des agences de presse publiées dans la rubrique "News" et les flashs d'information consultables depuis la rubrique "Vidéos" revêtent des formats ajustés à ce nouveau support, il n'en va pas de même pour les chaînes télévisées qui sont diffusées de la même manière que sur les grands écrans des télévisions. Si cette logique de diffusion multi-support permet aux utilisateurs de retrouver sur les écrans de leur téléphone mobile, les programmes qu'ils regardent habituellement à la télévision, il apparaît toutefois que les modes de réception diffèrent sensiblement suivant le support regardé. Par exemple, la réception sur les téléphones mobiles ${ }^{28}$ se déroule dans des lieux inhabituels tels que les bureaux ou les voitures; elle s'inscrit dans des interactions spécifiques: entre collègues ou entre passagers ; elle renouvèle le rapport aux contenus par la brièveté des créneaux d'utilisation ; elle modifie les formes d'écoute en contraignant les utilisateurs à accorder plus d'importance aux sons qu'aux images, etc.

Sur l'ensemble de ces traits qui caractérisent la réception des contenus multimédia sur les supports mobiles, nous nous contenterons de décrire ici les deux registres qui paraissent essentiels pour expliquer pourquoi les services multimédias $3 \mathrm{G}$ sont associés aux pratiques d'information. Le premier renvoie à la disponibilité du téléphone mobile en tant qu'il constitue un support médiatique qui suit les individus dans toutes les situations ${ }^{29}$. Le second concerne l'accessibilité des contenus dont les formats sont suffisamment attractifs pour former des opportunités d'usages propices au divertissement de nombreuses situations. Ces deux registres jouent un rôle important puisqu'ils s'inscrivent au principe des usages déployés dans toutes les situations : au domicile, sur le lieu de travail ou durant les trajets quotidiens.

Pour saisir la convergence entre ces deux registres, prenons l'exemple des individus qui regardent des chaînes télévisées dans les transports en commun. Les trajets quotidiens entre le domicile et le lieu de travail sont vécus comme des temps morts. De plus en plus d'individus se tournent alors

\footnotetext{
${ }^{28}$ O'HARA, MITCHELL, VORBAU, 2007

${ }^{29}$ LICOPPE, INADA, 2005.
} 
vers la multifonctionnalité de leur téléphone mobile afin de regarder des programmes télévisés. S'il leur est possible de déployer cet usage, c'est tout d'abord en raison de la disponibilité du mobile qu'ils gardent en permanence sur eux pour rester joignables. Mais ce n'est pas parce que ce nouveau support est continuellement à disposition que les usagers vont l'utiliser pour autant. Ils le font car ils considèrent que les contenus sont attractifs et qu'ils peuvent les consulter en quelques clics. Il devient alors agréable de se tourner vers son mobile pour regarder un bulletin d'information.

Il va de soi qu'un nombre conséquent d'utilisateurs, et plus particulièrement d'utilisatrices, vont préférer se tourner vers les séries qu'elles jugent bien plus divertissantes que les JT. Cependant, comme les chaînes télévisées ne proposent pas de séries le matin, elles sont contraintes d'attendre le trajet retour pour pouvoir les regarder. En début de journée, lorsqu'elles se rendent sur leur lieu de travail, elles se plient alors à la programmation télévisuelle et s'orientent majoritairement vers l'émission Télématin diffusée par France 2. C'est ainsi qu'en recherchant un programme distrayant, elles s'ouvrent à l'actualité du jour à travers les bulletins d'information proposés lors de cette émission. On peut croiser cette tendance avec la manière dont la radio alimente les pratiques d'information puisque les programmes radiodiffusés sont régulièrement entrecoupés de flashs info.

Il apparait également que le choix des contenus répond à la brièveté des opportunités d'usage qui excédent rarement dix minutes. En effet, comme le temps de chargement des chaînes télévisées prend une vingtaine de secondes et comme les trajets sont courts, les individus se fixent devant un programme sans recourir au zapping. Nous avons pu observer que la majorité des utilisateurs font alors en sorte de s'orienter vers des contenus qui peuvent être pris en cours de diffusion, et interrompus à tout moment, sans que cela ne soit problématique. A la différence des séries, les JT permettent ces rapides usages opportunistes dans la mesure où ils ne sont pas produits autour d'une structure d'intrigue. Ils peuvent être regardés quelques minutes, puis interrompus, sans que l'utilisateur éprouve la frustration de s'être arrêté en plein milieu de l'histoire. Il en va autrement des séries, et de l'ensemble des contenus produits autour d'une structure d'intrigue, puisqu'il peut être particulièrement frustrant de devoir cesser de les regarder lorsqu'on est arrivé à quai. Nous pouvons donc en déduire que ces usages ne répondent pas tant à des goûts affirmés (autour des séries, des films,...), que les individus aimeraient pouvoir reproduire à partir de leur téléphone multimédia, qu'à l'adaptation aux contraintes de la brièveté des créneaux d'utilisation.

Les usagers doivent plier leurs goûts à d'autres contraintes propres aux situations d'usages. Si un individu préfère regarder une série plutôt que de lire des dépêches, il peut être contraint de s'en satisfaire. Par exemple, la présence de nombreux passagers peut l'inciter à ne pas faire de bruit et le conduire à privilégier la lecture silencieuse des News ( $C f$. ci-dessous les captures d'écran d'un enregistrement vidéo effectué avec des lunettes caméra).

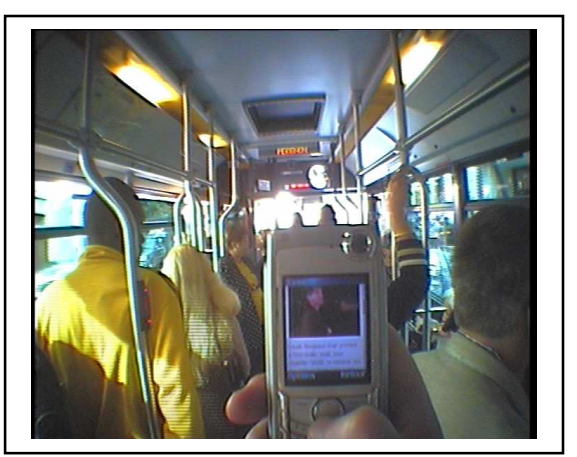

Debout, J.A. consulte les news écrites du WAP pour ne pas déranger les passagers du bus.

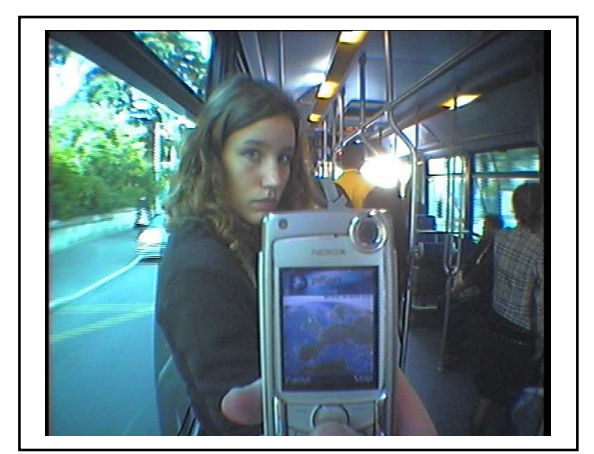

Une fois assis, J.A. n'éprouve plus de gêne à visionner la vidéo de la météo même si le son qui se propage attire l'attention d'une passagère. 
L'utilisation des services multimédias est donc à réinscrire sous la tension entre les stratégies des individus, visant à s'approprier les situations pour déployer des usages qui soient le plus possible en phase avec leurs attentes, et les contraintes de la situation qui délimitent l'horizon des usages possibles.

Ainsi, les individus associent les services multimédias à leurs pratiques d'information en raison de la disponibilité des téléphones mobiles et en raison de l'accessibilité de leurs contenus. Si nous mettons entre parenthèses les individus passionnés par les grilles sportives qui mobilisent ces services pour alimenter leur veille informationnelle, le choix d'utiliser son mobile pour s'informer ne répond pas tant à des goûts affirmés qu'à des ajustements vis-à-vis des situations d'usage. Ces ajustements se plient également à l'offre de contenu. Mais il faut bien considérer que l'essentiel ne se joue pas toujours au niveau des contenus regardés. Comme lorsqu'il est assis devant sa télévision, ce qui importe bien souvent au "mobinaute" qui se tourne vers ce nouveau support médiatique, c'est avant tout de rendre la situation plus agréable à vivre.

A partir de ces exemples paradigmatiques, nous avons abordé les usages respectifs de chacun des supports d'information mobilisés par les individus interrogés. Il s'est agi de décrire comment les modalités d'usages s'articulent au croisement entre l'horizon d'attente des usagers et la configuration des situations. C'est ainsi que l'écoute des informations radiodiffusées a été saisie à partir des situations qui permettent aux auditeurs de s'informer tout en remplissant de multiples tâches. La réception des journaux télévisés répond également à cette attente en s'articulant davantage avec les interactions familiales. En ce qui concerne l'engouement pour les journaux gratuits, il découle de leur distribution aux carrefours des trajectoires urbaines. Puisque ces contenus médiatiques sont culturellement peu valorisés, il est difficile de chercher à expliquer leur attrait à partir de leur légitimité culturelle. Il en va de même pour les "News" diffusées sur Internet. C'est bien leur inscription aux carrefours des navigations Internet qui leur confère une légitimité culturelle circonstancielle. C'est également le cas pour les services multimédias, consultables à partir des téléphones mobiles, qui voient la contrainte de la petite taille des écrans balayée par des motivations circonstancielles.

\section{CONCLUSION}

La diversification croissante des supports médiatiques permet aux individus de s'informer à partir d'une gamme étendue de sources d'information. Internet a par exemple déjà largement contribué à renouveler le paysage des pratiques d'information développées au domicile ou sur le lieu de travail. Aujourd'hui, la diffusion des téléphones mobiles de troisième génération vient modifier les modes de divertissements auxquels ont recours certains passagers des transports en commun. Cette tendance pose donc la question de la problématique articulation entre les usages successifs des différentes sources.

A travers cette contribution, nous avons tenté d'y apporter quelques éléments de réponse en partant du principe que les pratiques d'information ne peuvent plus être abordées de manière unifiée. Il est difficile de donner sens à leur distribution sur des supports différents, dans des situations variées, sous la seule perspective consistant à croiser les profils sociodémographiques des individus avec la légitimité culturelle des sources. En effet, même les habitudes médiatiques qui réactualisent au jour le jour les plus forts enjeux identitaires, comme par exemple le choix d'un quotidien en fonction de sa coloration politique, plient sous les effets de disponibilité de la presse gratuite. Sans chercher à stigmatiser cette évolution, nous avons tenté d'en dévoiler quelques ressorts.

Pour cela, nous venons de proposer une lecture pragmatique des pratiques d'information en décrivant comment elles sont rarement déployées pour tendre vers un but défini autour des informations elles-mêmes, comme lors des recherches d'informations. Elles répondent plutôt à 
des motifs pragmatiques définis en situation. En conclusion, nous proposons de les regrouper sous trois registres de propension à prendre en considération pour saisir comment, en fonction de la configuration des situations quotidiennes, s'opère la sélection des sources d'information et les usages qui en sont faits. Ces propensions sont fonction des opportunités de la situation, celles formées par les personnes en présence, les activités réalisées, les supports à disposition et, plus généralement, l'environnement ambiant.

Le premier registre de propension est celui de la multi-activité et des types d'interactions. Si ce registre est caractéristique de la réception des JT et des émissions de radio, il s'applique aussi aux autres supports d'information. Par exemple, si la décision de lire un quotidien ne parait pas directement liée à ce registre, elle l'est indirectement : un individu va préférer ouvrir un journal quand il est seul et qu'il n'est pas engagé dans une autre activité.

Le deuxième registre concerne le degré de disponibilité du support d'information qui est fonction de la configuration de la situation. Ainsi, l'attractivité des téléphones mobiles repose sur leur continuelle disponibilité, notamment en situation de mobilité et sur le lieu de travail. Cette dimension du degré de disponibilité des supports d'information est indissociable de la dimension de l'accessibilité des contenus qu'ils véhiculent, c'est-à-dire de la rapidité avec laquelle ils vont donner accès à l'information recherchée ou au divertissement espéré. Ce registre est d'autant plus déterminant que les temps interstitiels sont vécus dans l'urgence. C'est ainsi que la contrainte du détour par les kiosques peut participer au désaveu de la presse payante.

Le troisième registre est quant à lui relatif à l'attractivité des formats médiatiques et à l'économie cognitive. La sélection d'un média va dépendre du degré d'attention que va solliciter son usage, selon le format des informations qu'il véhicule (écrites, orales ou télévisuelles). Cette sollicitation des formats médiatiques est variable d'une situation à l'autre ${ }^{30}$ suivant le lieu, le moment de la journée et les personnes en présence. C'est sous les effets de ce registre que l'argument de la gratuité des quotidiens perd de sa force face à l'attractivité des chaînes télévisées consultables sur les téléphones mobiles.

A la différence des travaux sur les "contextes d'écoute" ${ }^{31}$ qui se focalisent avant tout sur le déroulement de la réception, ces registres permettent également de remonter en amont au niveau de la sélection des supports médiatiques. Ils viennent ainsi compléter les recherches qui situent cette sélection au centre de leurs analyses en croisant les profils sociodémographiques des individus avec la légitimité culturelle des sources d'information ${ }^{32}$. Cette complémentarité prend tout son sens lorsque les effets structurants de la légitimité plient sous les opportunités des situations, comme c'est par exemple le cas à chaque fois qu'un individu va préférer, au détour par un kiosque, attraper le quotidien gratuit qu'un distributeur lui tend.

En proposant ces trois registres, nous tentons d'appréhender la sélection des sources d'information de manière endogène en la situant au niveau des différentes prises des situations quotidiennes qui co-orientent les individus vers un mode spécifique de consommation médiatique. En procédant ainsi, nous décrivons comment les habitudes médiatiques ne sont pas tant renouvelées par les nouveaux supports d'information que par la possibilité d'inscrire et de se stabiliser leurs usages dans les situations quotidiennes, que ce soit au domicile, en mobilité ou sur le lieu de travail. Ainsi, nous proposons de croiser ces registres pour déconstruire ces formes d'inscription qui sont tout autant co-déterminées par les personnes en présence et les activités réalisées en parallèle des usages que par l'attractivité du support et sa disponibilité dans une situation précise.

\footnotetext{
${ }^{30}$ LULL, 1990.

${ }^{31}$ MORLEY, 1986.

${ }^{32}$ LAHIRE, 2004b, 2004a, 1998.
} 


\section{REFERENCES}

BEAUDOUIN V., BEAUVISAGE T., CARDON D., VELKOVSKA J. (2003), "L'entrelacement des médias dans la constitution des publics de Loft Story", Rapport FT R\&D/ 8019.

BEAUDOUIN V., FLEURY S., PASQUIER P., HABERT B., LICOPPE C. (2002), "Décrire la toile pour mieux comprendre les parcours. Sites personnels et sites marchands", Réseaux, $\mathrm{n}^{\circ} 116$, p. $199-225$.

BEAUVISAGE T. (2004), Sémantique des parcours des utilisateurs sur le Web, Thèse de doctorat, Paris X. CERTEAU (DE) M. (1980), L'invention du quotidien. 1. Arts de faire, Gallimard, Folio Essais, 1990.

CHARAUDEAU P. (1997a), Le discours d'information médiatique. La construction du miroir social, Nathan, Paris, 1997.

CHARAUDEAU P. (1997b), "Les conditions d'une typologie des genres télévisuels d'information", Réseaux, $\mathrm{n}^{\circ} 81$.

CONEIN B., JACOPIN E. (1994), "Action située et cognition : le savoir en place", Sociologie du travail, n'XXXVI, 4/94, Dunod, p. 475-500.

CORNER J. (1991), "Meaning, genre and context: the problematics of "public knowledge" in the audience studies", in Curran J. and Gurevitch M. (eds), Mass media and society, Edward Arnold, London.

KIRSH D. (1995), "The Intelligent Use of Space", in Artificial Intelligence, Vol. 73, Number 1-2, pp. 31-68.

LAHIRE B., (2004a) "Individu et mélanges des genres", Réseaux, n 126, p.89-111.

LAHIRE B. (2004b) La culture des individus. Dissonances culturelles et distinction de soi, Edition de La découverte, Paris.

LAHIRE B. (1998), "Matrices disciplinaires de socialisation et lectures étudiantes", Bulletin des bibliothèques de France, ${ }^{\circ}$ 5, p.58-61.

LICOPPE C., INADA Y. (2005), "Les usages émergents d'un jeu multijoueurs sur terminaux mobiles géolocalisés. Mobilités équipées dans un japon "augmenté" et "rencontres d'écran", Réseaux, n¹33, p. 135164.

LULL J. (1990), Inside Family Viewing. Ethnographic Research on Television's Audiences, Londres, Routledge (Comedia).

MORLEY D. (1986), Family Television: Cultural Power and Domestic Leisure, London: Comedia.

MORLEY D., SILVERSTONE R. (1990), Domestic Communications: technologies and meanings, Media, Culture and Society, 12 (1): 31-55.

MUZET D. (2006), La mal info : enquête sur des consommateurs de médias, L'aube.

OGIEN A. (1989), "La décomposition du sujet", in Joseph I. (dir.), Le parler frais d'Erving Goffman, Paris, Minuit.

O'HARA K., MITCHELL A.S., VORBAU A. (2007), "Consuming Video on Mobile Devices", in Proceedings of CHI '07, April 28 - May 3, 2007, San Jose, California, USA.

PHARABOD A. S. (2004a), Les terminaux domestiques et leurs usages : une ethnographie des foyers multi-équipés, Rapport FT R\&D, n 8664.

PHARABOD A. S. (2004b), "Territoires et seuils de l'intimité familiale. Un regard ethnographique sur les objets multimédias et leurs usages dans quelques foyers franciliens", Réseaux, nº123, p. 85-111.

PROULX S. (2002), "Trajectoires d'usages des technologies de communication : les formes d'appropriation d'une culture numérique comme enjeu d'une "société du savoir" in La Vega (De) J., Licoppe C., "Usages émergents des TIC", Annales des télécommunications.

PROULX S., LABERGE, M.-F. (1995), "Vie quotidienne, culture télévisuelle et construction de l'identité familiale", Réseaux, nº70, p.121-140 
QUERE L. (1999), "Action située et perception du sens", in Fornel (De) M., QUERE L. (dir.), La logique des situations. Nouveaux regards sur l'écologie des activités sociales, Paris, Éditions de l'École des Hautes Études en sciences sociales.

RELIEU M., OLSZEWSKA, B. (2004), "La matérialisation d'Internet dans l'espace domestique : une approche située de la vie domestique", Réseaux, n 123, p.119-149.

SEMPRINI A. (1994), L'information en continu. France Info et CNN, Paris, Nathan, 1997.

SUCHMAN L. A. (1987), Plans and situated actions - The problem of human-machine communication. Cambridge: Cambridge University Press.

TAYLOR A. S., HARPER R. (2002), "Switching on to switch off: a analysis of routine TV watching habits and their implications for electronic programme guide design", in usableiTV, 1(3), 7-13. 